\title{
RECAI
}

RECAI Revista de Estudios en Contaduría,

Administración e Informática

ISSN: 2007-5278

danielarturoc@yahoo.com.mx

Universidad Autónoma del Estado de México

México

\section{Evaluación de los factores que influyen sobre la percepción de los empleados en cuanto a la situación de responsabilidad social de una empresa manufacturera}

Sánchez López, Eduardo; González Espinoza, Mayda; González Larrinaga, Lucio Alberto

Evaluación de los factores que influyen sobre la percepción de los empleados en cuanto a la situación de responsabilidad social de una empresa manufacturera

RECAI Revista de Estudios en Contaduría, Administración e Informática, vol. 9, núm. 24, 2020

Universidad Autónoma del Estado de México, México

Copyright 2019 Universidad Autónoma del Estado de México

Esta obra está bajo una Licencia Creative Commons Atribución-NoComercial-SinDerivar 4.0 Internacional. 


\section{Evaluación de los factores que influyen sobre la percepción de los empleados en cuanto a la situación de responsabilidad social de una empresa manufacturera}

Evaluation of the factors that influence the perception of employees regarding the social responsibility situation of a manufacturing company

Eduardo Sánchez López

Universidad Autónoma de Baja California, México

edsanmxl@hotmail.com

Mayda González Espinoza

Universidad Autónoma de Baja California, México

mayda.gonzalez@uabc.edu.mx

Lucio Alberto González Larrinaga

Universidad Autónoma de Baja California, México

lucio.alberto.gonzalez.larrinaga@uabc.edu.mx

Recepción: 02 Septiembre 2019

Aprobación: 02 Diciembre 2019

\section{Resumen:}

Con el fin de definir qué factores influyen sobre la percepción de la responsabilidad social que tienen los empleados de una empresa manufacturera, se llevó a cabo una encuesta a una muestra representativa de empleados. Para ello, se utilizó un instrumento de once dimensiones formateado en una escala Likert. La información obtenida fue evaluada mediante un modelo de regresión logística binaria, en el cual la variable dependiente fue la percepción del nivel de responsabilidad social de la empresa. Las variables independientes fueron: igualdad laboral, atención a empleados con capacidades diferentes, condiciones de trabajo, derechos laborales, ambiente de trabajo, cuidado del medio ambiente, uso de agua, ética, respeto a propiedad intelectual, aplicación de leyes y reglamentos, así como el sexo del entrevistado. La estimación del modelo resultó en que solo las variables: ambiente de trabajo, uso de agua y cumplimiento de leyes y reglamentos resultaron significativas $(\mathrm{p}<.05)$; todas ellas presentaron un OR superior a 1.5 . La significancia de tan solo tres variables indica la necesidad de que la empresa mejore su situación de responsabilidad social.

Palabras Clave: Responsabilidad social corporativa, empleados, percepción.

\section{Abstract:}

To determine the variables that influence the social responsibility perception of a manufacturing firm's employees, a survey was conducted with a representative sample of such employees. To do so, a survey including responses formatted in a Likert-type scale was conducted. The data were analyzed by running a binary-logistic regression model that included the perception of the employees regarding the firm's social responsibility as a dependent dichotomous variable. The independent variables in the model were: labor equality, attention to employees with special needs, work conditions, labor rights, work environment, environmental care, water use, ethics, intellectual property protection, compliance with laws and regulations, and, of course, the respondent's gender. Once the model was estimated, it was found that only the variables: work environment, water use and compliance with laws and regulations were statistically significant $(\mathrm{p}<.05)$; each of these variables with OR values above 1.5 . The significance level of only three out of twelve variables indicates that the firm must improve its social responsibility situation.

KEYWORDS: Corporate social responsibility, employees, perception.

\section{INTRODUCCIÓN}

Ser socialmente responsable es una característica de los individuos que apoya el buen desarrollo de la sociedad. Sin embargo, este comportamiento no está limitado al individuo, Amdani (2017), afirma que responsabilidad social es el compromiso contraído por las acciones u omisiones de cualquier individuo o grupo que genera un 
impacto en la sociedad y en su comunidad vecina. Por lo tanto, se requiere del comportamiento responsable de todos los integrantes de una comunidad incluyendo las empresas.

En la empresa la responsabilidad social cobra una importancia especial dada la interacción que en múltiples aspectos tiene con la sociedad. De acuerdo con lo expresado por Thompson (2012), la esencia del comportamiento de negocios responsable con la sociedad es que una empresa debe equilibrar las acciones estratégicas para beneficiar a sus accionistas con el deber de ser un buen ciudadano corporativo. Considerando esta definición, es necesario un compromiso de entre las empresas y la sociedad y hace que las empresas deban comportarse como ciudadanos responsables, con apego a la ética corporativa y respetando tanto a las personas como al medio ambiente. Por lo que la empresa no solamente tiene una función económica, sino también obligaciones de tipo ético con respecto a la sociedad" (Soto y Cárdenas, 2007).

De acuerdo con Avendaño (2013) las empresas se inclinan a respaldar principalmente la educación y salud, y en un segundo lugar, arte, cultura y medioambiente. Esto pudiera considerarse como negativo ya que la obligación extraeconómica que la empresa tiene con la sociedad no debe basarse solo en acciones de apoyo a las causas sociales como hacer obras caritativas y donaciones en especie o monetarias, la empresa con verdadera responsabilidad social debe principalmente realizar acciones en las áreas laboral, ambiental, ético y normativo que generen la confianza y respeto de los ciudadanos.

Guerrero et al., (2006) consideran que el desarrollo cultural de los trabajadores promueve la humanización de los procesos y afecta las relaciones sociales, por lo que deben establecerse mejores condiciones para el desempeño de su actividad laboral con el fin de incrementar su calidad de vida laboral. Una empresa con un nivel adecuado de responsabilidad social responde de mejor manera a estas exigencias.

Dado que la Responsabilidad Social Corporativa (RSC) busca el bien común de los grupos de interés de una organización, mediante la implementación de prácticas internas y externas (Nieto, Melgar y Coronel, 2015), entonces es importante para la empresa implementar una política eficiente de responsabilidad social ya que su seguimiento puntual puede afectar positivamente a la organización en una diversidad de indicadores entre los cuales destacan la mejora de la imagen de la empresa, la generación de lealtad de los trabajadores, la atracción de personal más calificado, una mayor disposición a colaborar en situaciones difíciles, la captación de inversiones estables (Raufflet, et al., 2012).

En cuanto a los aspectos laborales asociados a la RSC se encuentran: remuneración y prestaciones, seguridad y salud, derechos laborales y normas de trabajo globales, trato justo y ético en la contratación, revisiones, promociones y áreas afines (Hellriegel, et al., 2017). Todos estos elementos son muy relevantes para el desempeño de los trabajadores, por lo que esta relación toma una relevancia importante para la empresa.

Es importante destacar que la RSC es regulada directa o indirectamente por instrumentos internacionales y legislación nacional y el incumplimiento de la política es sancionado tanto administrativa como judicialmente, pero también el incumplimiento de los estándares de RS resulta en una mala reputación para las empresas infractoras (Anguita, 2011).

En un estudio de responsabilidad social realizado por Barrosso (2008) se obtuvo que para las empresas el ser socialmente responsable es mejorar las condiciones del trabajador y de la comunidad donde operan, el cuidado y preservación del medio ambiente y el dar donativos. Si las empresas practican la responsabilidad social en el sentido amplio del concepto, las ganancias serán el resultado de su compromiso hacia todos y la sociedad, serán la recompensa de su compromiso social, lo cual ha sido posible en empresas de gran éxito y que son socialmente responsables.

De acuerdo con Kotler y Lee (2005), las empresas que adoptan la Responsabilidad Social Corporativa (RSC) incrementan sus ventas, mejoran su participación en el mercado, posicionan mejor su marca e imagen corporativa, y con ello atraen y retienen mejores empleados, pero también la RSC promueve la disminución de los costos operativos y atrae la inversión. Adicionalmente una empresa que se posiciona como socialmente responsable mejora su reputación y tomando en cuenta que otros investigadores consideran que la reputación 
se ha convertido en un activo importante y un objetivo empresarial (Schwartz y Gibb 2000), entonces las empresas buscan alcanzar esta característica de responsabilidad en apoyo a una percepción positiva por parte de la sociedad.

Considerando lo expresado por Randstad (2015), en cuanto a que: "El ambiente laboral es uno de los elementos más importantes en el día a día de la empresa, sin embargo, las características que lo determinan son difíciles de precisar. Pueden ser de naturaleza tangible o intangible, y se ha demostrado que influyen notoriamente en la productividad y en la vida privada de los empleados." Y considerando que la RSC tiene una intensa relación con los aspectos laborales, entonces surge como un elemento de alto interés para las compañías la percepción que sus empleados tienen en cuanto a la situación de la RSC en su empresa.

Debido a lo previamente expresado el presente trabajo tiene como objetivo definir qué factores influyen sobre la percepción que tienen los empleados sobre la situación de responsabilidad social de una empresa manufacturera.

\section{Metodología:}

Con el fin de definir los factores que influyen sobre la percepción que tienen los empleados de una empresa manufacturera sobre la situación que esta presenta sobre la responsabilidad social, ubicada esta empresa en la ciudad de Mexicali Baja California, se llevó a cabo la selección de una muestra estadística a partir de la población de estos elementos $(\mathrm{N}=70)$. La muestra se obtuvo considerando una confianza de .95 y un error máximo de .05, utilizando la siguiente fórmula (Torres et al., 2006):

$$
n=\frac{\mathrm{N} * \mathrm{Z} \alpha / 2 * \mathrm{p} * \mathrm{q}}{\mathrm{d}^{2} *(\mathrm{~N}-1)+\mathrm{Z} \alpha / 2 * \mathrm{p} * \mathrm{q}}
$$




\author{
Donde: \\ $\mathrm{n}=$ tamaño de muestra \\ $\mathrm{N}=$ Tamaño de la población \\ $\mathrm{Z} \alpha /_{2}=$ Valor crítico \\ $\mathrm{p}=$ Probabilidad de éxito \\ $q=$ probabilidad de fracaso \\ $d=$ error
}

El cálculo de la muestra dio por resultado aplicar 61 encuestas. Cabe mencionar que se aplicaron y analizaron 4 encuestas más que las que arrojó la fórmula, con un total de 65 .

Mediante una revisión bibliográfica se realizó la operacionalización de las variables. Se definieron dos variables, la responsabilidad social y la percepción:

TABLA 1

Operacionalización de las variables

\begin{tabular}{|c|c|c|}
\hline \multicolumn{3}{|l|}{ Marco Teórico } \\
\hline Variable & Dimension & Indicador \\
\hline Responsabilidad social & Empleados. Laasch y Conaway (2015). & Igualdad laboral. Lemus (2009). \\
\hline \multirow[t]{15}{*}{ Según Turker (2009) } & & Atención a empleados con capacidades differentes. Dalton, Hoyle, y Watts (2007). \\
\hline & & Atención Medica. Morris, Bohlandery Snell (2017) \\
\hline & & Compensación. Mondy (2010). \\
\hline & & Entorno fisico en buenas condiciones y seguridad. Dessler (2009). \\
\hline & & Derechos laborales. Mondy (2010). \\
\hline & & Trato justo en la contratación. Morris, Bohlander Y Snell (2017). \\
\hline & & Salud mental. Oblitas (2017). \\
\hline & & Ambiente laboral. Riquelme (2017). \\
\hline & Medio ambiente. Collier (2016). & Reciclaje. Vesilind, Morgan y Heine (2013). \\
\hline & & Manejo de residuos. Romero, Romero y Muñoz (2015). \\
\hline & & Uso eficiente de agua. Campaña, Diaz, y Mendoza (2011) \\
\hline & Ética. Arbaiza (2014). & IIntegridad. Según Lussier (2016). \\
\hline & & Respeto a propledad intelectual. Debeljuh (2009). \\
\hline & Legal. Williams (2013). & Aplicación de Leyes y reglamentos. The World Bank (2006). \\
\hline & Mercadotecnia Interna. Ahmed y Rafiq (2003). & Comunicación interna.Chiavenato (2007) \\
\hline
\end{tabular}

Para recopilar la información relevante se construyó un instrumento el cual consideró 11 variables independientes: igualdad laboral ( 5 afirmaciones), atención a empleados con capacidades diferentes ( 4 afirmaciones), entorno de trabajo ( 4 afirmaciones), derechos laborales ( 3 afirmaciones), ambiente de trabajo ( 6 afirmaciones), cuidado del medio ambiente ( 8 afirmaciones) y uso de agua ( 3 afirmaciones), ética ( 4 afirmaciones), respeto a propiedad intelectual ( 4 afirmaciones) aplicación de leyes y reglamentos ( 4 afirmaciones) y sexo del entrevistado. A los entrevistados se les dio una serie de afirmaciones, como, por ejemplo: "La empresa ofrece iguales oportunidades a todos los empleados", y se les solicito lo siguiente: “Califica del 1 al 10”. A excepción del reactivo de sexo del entrevistado que presentó una escala binomial, el resto de las afirmaciones fueron contestadas mediante de la selección de una respuesta en una escala 
RECAi Revista de Estudios en Contaduría, Administración e Informática, 020, 9(24), Enero-Abril, I...

de Likert cuyo rango consideró diez puntos. Para evaluar la respuesta de los entrevistados el instrumento conformado utilizó la escala con un rango desde (1), siendo esta la calificación más baja, y finalizó con (10), siendo esta la calificación más alta.

Los resultados de las respuestas a los reactivos en escala Likert de cada variable fueron utilizados

para obtener un promedio y esos valores utilizados como valores para la estimación del modelo.

Adicionalmente se evaluó la percepción de la situación (variable dependiente) utilizando la afirmación: "La empresa donde trabaja actúa de manera socialmente responsable”. Esta afirmación presentó las mismas características de escala como las anteriores, pero fue dicotomizada de acuerdo con el siguiente criterio: Si el valor de la respuesta fue superior a 8 , le fue asignado el valor de 1 , cualquier respuesta igual o menor de 8 recibió el valor de 0 .

Con el fin de hacer una prueba piloto del instrumento y realizar los ajustes necesarios en cuanto a su aplicación se seleccionaron 30 individuos de la población. Adicionalmente la prueba permitió realizar una prueba de fiabilidad utilizando el alfa de Cronbach.

Debido a que se buscó estimar la relación que existe entre una variable dependiente de tipo dicotómico y un grupo de variables independientes se seleccionó un modelo de regresión logística binario el cual presenta un diseño para describir una probabilidad. Por lo que la probabilidad a modelar puede ser expresada con la siguiente notación (Kleinbaum y Klein, 2010):

$$
P\left(D=1 \mid X_{1}, X_{2}, \ldots, X_{k}\right)
$$

Siendo el modelo a estimar el siguiente:

$$
P\left|X=\frac{1}{1+e^{-(\alpha+\Sigma \beta i X i)}}\right|
$$

$\alpha$ y $\beta$ i son parámetros desconocidos.

La regresión logística permite obtener una razón de probabilidades calculada como: $\mathrm{Pi} / 1-\mathrm{Pi}$, siendo $\mathrm{Pi}$ la probabilidad de que se presente el evento de interés, por lo que su cálculo permite estimar qué tan más probable es que se presente el evento, dado determinado valor o presencia de una variable. (Asteriou y Hall, 2011). 


\section{Resultados}

Para la población de 70 empleados en la empresa, el cálculo de la muestra nos dio por resultado aplicar 60.77 encuestas. Se hicieron 65 entrevistas, ya que las 5 personas restantes no estuvieron disponibles o no estaban en la empresa en las dos ocasiones que visitamos la empresa para hacer entrevistas.

El resultado de la prueba piloto realizada al instrumento indico que no era necesario hacer ajustes.

Con excepción de las variables igualdad laboral, ambiente de trabajo y derechos laborales que obtuvieron valores de alfa de Cronbach de $.87, .85$ y .88 respectivamente, el resto de las variables alcanzaron valores superiores a .90 por lo que se consideró que el instrumento presentó un buen nivel de fiabilidad.

TABLA 2.

Alfa de Cronbach por constructo

\begin{tabular}{|c|c|}
\hline Construto & Alfa de cronbach \\
\hline Igualdad laboral & 0.871 \\
\hline Atención a emple ados con capacid ades differentes & 0.928 \\
\hline Átendión Medica & 0.970 \\
\hline compensación & 0.889 \\
\hline Entorno fisico en buenas condicicones y seguridad & 0.927 \\
\hline Derechos Itaborales & 0.882 \\
\hline Treto justo en la contratación & 0.900 \\
\hline Solud mental & 0.897 \\
\hline Ambiente laboral & 0.854 \\
\hline Reccligla & 0.956 \\
\hline Man ejo de Residuos & 0.922 \\
\hline Uso eficiented de agua & 0.916 \\
\hline Integridad & 0.892 \\
\hline Respeto a propiedadi intelectual & 0.934 \\
\hline Apicicción de leyes y reglamentos & 0.908 \\
\hline comunicacín intema & 0.887 \\
\hline
\end{tabular}

Debido a que la estimación del modelo inicial de regresión de regresión logística (11 variables independientes) resultó en valores altos de P para las variables: igualdad laboral, atención a empleados con capacidades diferentes, condiciones de trabajo, derechos laborales, cuidado del medio ambiente, ética, respeto a propiedad intelectual, y sexo del entrevistado, mientras que las variables: ambiente de trabajo, uso de agua, aplicación de leyes y reglamentos presentaron valores bajos de P. Se conformó un segundo modelo a estimar, el cual resultó con valores de significativos de $\mathrm{P}(\mathrm{P}<.05)$ para cada una de estas tres variables. Los resultados de la estimación se presentan en la tabla 2. 
TABLA 2.

Resultados de la estimación del segundo modelo.

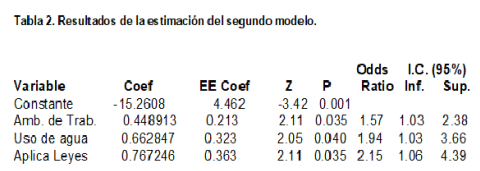

La Prueba de bondad de ajuste de Hosmer- Lemeshow presentó un valor de $\mathrm{P}=0.14$ por lo que las predicciones de las probabilidades presentan una desviación con respecto a las observadas que respalda el poder predictivo de la distribución binomial.

Como es posible observar en la tabla 2, los valores de los coeficientes correspondientes a las variables dependientes ambiente de trabajo, uso de agua, aplicación de leyes y reglamentos presentan signos positivos, por lo es posible asumir que los incrementos en cada una de las variables afectan positivamente la apreciación de la responsabilidad social.

Adicionalmente, la tabla 2 muestra los valores tanto puntuales como de intervalo de la razón probabilidades también reportada como odds ratio (OR) que corresponde a cada una de las variables independientes. Como es posible observar en los tres casos el OR es superior a 1 y si bien en el límite inferior del intervalo de los tres casos se observan valores ligeramente superiores a 1 , es importante destacar que los límites superiores indican que, por ejemplo, sea posible observar que un incremento unitario en la calificación que da un empleado a la aplicación de leyes y reglamentos de la empresa hace 4.39 veces más probable que este considere a la empresa como socialmente responsable. En el caso de las variables ambiente de trabajo y uso de agua pueden ser 2.38 y 3.66 veces más probables. Considerando que el objetivo del presente trabajo es definir los factores que influyen en la percepción de los empleados además de la probabilidad de ocurrencia, la significancia estadística de las tres variables independientes permite que se considere que estos factores tienen una influencia sobre la percepción de los empleados respecto la responsabilidad social de la empresa y por lo tanto el comportamiento de esta última puede ser importantemente explicada por el trío de variables independientes significativas, ya que el modelo de regresión logística permite realizar una predicción de la variable dependiente, que en el caso de este estudio indica la probabilidad de que el entrevistado perciba a la empresa como socialmente responsable. (Kennedy, 1998).

\section{Discusión}

Algunos de los hallazgos de este estudio son inesperados, destacando el caso de la nula relación encontrada entre responsabilidad social de la empresa y el cuidado del medio ambiente ya que considerando lo reportado por investigaciones en los Estados Unidos (Pride et al., 2015) en cuanto a que los ciudadanos esperan que tanto los líderes de negocios, como los funcionarios gubernamentales emprendan acciones decididas a limpiar el medio ambiente y mantenerlo en buenas condiciones.

Por otra parte, otros investigadores (Aguilera y Puerto, 2012) han reportado que el efecto ambiental se considera como un elemento constitutivo de la actividad empresarial. Los resultados contradictorios a la literatura existente pueden ser explicados por el hecho que el grupo de interés no fue un agente externo a la empresa, sino uno interno, como lo son los empleados, y para ellos el elemento medioambiental no 
tiene relevancia. Además, este comportamiento puede también ser explicado por lo expresado en otras investigaciones en las que se encontró que los diferentes agentes presentan una diversidad de perspectivas respecto a la responsabilidad social empresarial (Amir y Amir y Rubín, 2010). Sin embargo, si se considera que la variable uso del agua resultó significativa, entonces es posible pensar que la situación ambiental tuvo alguna relevancia para la percepción de la responsabilidad social empresarial.

El resultado de la variable cuidado del medio ambiente es útil para establecer las diferencias en las percepciones de los diferentes agentes de interés, pues si bien, en el presente estudio no tuvo relevancia, se ha reportado que para el consumidor una de las acciones que realiza la empresa que más asociadas están a un comportamiento socialmente responsable, representan a la dimensión medioambiental (Bigné et al, 2005).

El hecho de que el presente trabajo haya considerado a las percepciones de los empleados como fuentes de información, y tomando en cuenta que estudios como los de Avendaño (2013) considera a las variables: mejora de las condiciones laborales y respeto de las normas laborales como indicadores de responsabilidad social, es posible explicar la relevancia tanto estadística como práctica de las variables: ambiente de trabajo y aplicación de leyes y reglamentos. De estas dos variables, considerando los valores puntuales y de intervalo de la razón de probabilidades, sin duda destaca como más relevante para influir en la percepción de los empleados la segunda. Por lo que la empresa debe aprovechar la situación que prevalecía en el momento de la realización de este estudio para mantener un buen nivel de aplicación y seguimiento de las leyes y reglamentos que le son relevantes.

Otro elemento que explica el comportamiento de las dos variables asociadas al ambiente laboral es lo reportado por Guerrero et al (2006) respecto a que la calidad de vida en el trabajo es un conjunto de creencias que incluyen los esfuerzos por aumentar la productividad y mejorar la moral de las personas, con énfasis en la participación de la gente, la preservación de su dignidad, eliminando los aspectos disfuncionales de la jerarquía organizacional. Si bien se ha reportado que en las pequeñas y medianas empresas la calidad laboral es la principal área donde establecen sus esfuerzos pues la facilidad de implementación y las relaciones estrechas que desarrolla con el empleado, genera intuitivamente un comportamiento de apoyo y respeto a los derechos, y considerando que las empresas grandes han adoptado de mejor manera a la responsabilidad social (López, 2013), es posible pensar que la empresa en la que se desarrolló el estudio ha tenido buen éxito en cuanto a la percepción que tienen sus empleados respecto a la implementación de política de responsabilidad social.

Sin embargo, a pesar de lo anteriormente planteado, esto no asegura un comportamiento socialmente responsable ya que las variables no laborales no arrojaron resultados que respalden este comportamiento. No obstante, se debe de tomar en cuenta de que estos factores son mejor evaluados por agentes externos que por los empleados.

Un resultado de alta relevancia es el observado en la variable ética ya que si se considera lo expresado por Chirinos et al. (2012) en cuanto a que todas las decisiones organizacionales deben sustentarse en lo ético, el hecho de que esta dimensión no haya sido relevante para influir en la percepción de la responsabilidad social puede significar que el empleado desestima un valor de fundamental importancia en la responsabilidad social, esto no implica que la empresa no presente un comportamiento ético, ya que si se toma en consideración que de acuerdo a Garriga y Melé (2004) en cuanto a que las teorías de la responsabilidad social corporativas incluyen como elemento fundamental el que la empresa contribuya a conformar una mejor sociedad haciendo lo que es éticamente correcto, el hecho de que se haya encontrado una relación positiva entre las variables independientes de este modelo y la percepción de la responsabilidad social respalda de alguna manera la ética corporativa.

De acuerdo con León (2008), la RSC se está convirtiendo en el camino a seguir porque las nuevas tendencias del comportamiento del consumidor exigen que así sea. Además, un comportamiento responsable, lejos de ser un gasto no retornable debe ser considerado como una inversión social que trae a las empresas, entre otros, beneficios tales como: aumenta el compromiso de los empleados, si ellos se involucren 
en las causas sociales y éstas a su vez estén consideradas en la estrategia corporativa (Guédez, 2006) y mejora las condiciones de trabajo (menor ausentismo y rotación laboral, y más calidad y productividad).

La responsabilidad social empresarial debe ser vista como una inversión ya que se ha reportado que el compromiso organizacional del empleado se incrementa conforme aumenta la percepción de que su empresa es socialmente responsable (Turker, 2009) y si bien es cierto que en este estudio la percepción de los empleados sobre la responsabilidad social fue influida por tres variables, el hecho de que nueve variables evaluadas no resultaron significativas indica la necesidad de que la empresa mejore su situación, mediante una estrategia tanto interna como externa que incida positivamente sobre cómo los agentes perciben a la empresa.

\section{Conclusiones}

Tomando en consideración que la literatura reporta una relación positiva entre las ocho variables que no resultaron estadísticamente significativas, el hecho de haber sido este comportamiento hace necesario revisar con mayor detalle la situación que guardan estos elementos de responsabilidad social dentro de la empresa buscando su mejora, de tal forma que lleguen a ser ubicados por los empleados en niveles altos y se conviertan en elementos de apoyo en la mejora de la responsabilidad que la empresa tiene con la sociedad y su entorno.

En cuanto a las variables ambiente de trabajo, uso de agua, aplicación de leyes y reglamentos al resultar relevantes para influir en la percepción de los empleados sobre la responsabilidad de su empresa se convierten en factores importantes para apoyar esta percepción positiva, por lo que la gerencia de la empresa debe revisar de manera continua su situación buscando que sirvan como indicadores de la responsabilidad social. En caso de observarse cambios negativos en estos indicadores de la percepción, realizar un diagnóstico y tomar las medidas más convenientes para solucionar el problema.

La selección del grupo de interés es importante para evaluar la percepción del nivel de responsabilidad empresarial. La visión de lo que es socialmente responsable no es igual en un empleado de la empresa que para otro grupo externo, pero considerando que, en el caso de los empleados, una percepción positiva de esta característica empresarial puede motivar al trabajador a hacer mejor su trabajo, entonces resulta muy conveniente para la empresa evaluar esta perspectiva y dependiendo de los resultados hacer los ajustes necesarios, pues esto puede traducirse en un beneficio económico.

\section{ReFERENCIAS}

Aguilera, A. y Puerto, D. P. (2012). Crecimiento empresarial basado en la responsabilidad social. Pensamiento \& Gestión, 32, 1-26.

Amdani, Y. (2017). La Responsabilidad Social, un Compromiso de todos. Empresas \& Management

Amir, B. y Amir R. Corporate social responsibility as a conflict between shareholders Journal of Business Ethics, 97(1), 71-86.

Anguita, C. (2011). El rol del derecho en la responsabilidad social de la empresa. Ars Boni et Aequi, 8 (1), 215-233.

Asteriou, D. y Hall, G. (2011) Applied Econometrics. Second Edition. Londres, RU: Palgrave Macmillan.

Avendaño, C. y William, R. (2013). Responsabilidad social (RS) y responsabilidad social corporativa (RSC): una nueva perspectiva para las empresas. Revista Lasallista de Investigación. 10(1), 152-163.

Barroso, F.G. (2008). La responsabilidad social empresarial. Un estudio en cuarenta empresas de la ciudad de Mérida, Yucatán. Contaduria y Administración, 226, 73-91.

Bigné, E., Chumpitaz, R., Andreu, L. y Swaen, V. (2005). Percepción de la responsabilidad social corporativa: Un análisis cross-cultural. Universia Business Review, 5, 14-27. 
Chirinos, M.E., Fernández, L.y Sánchez, G. (2012). Responsabilidad empresarial o empresas socialmente responsables. Razón y Palabra, 81, Disponible en http://www.redalyc.org/articulo.oa?id=199524700002 Consultado el 21 de agosto de 2019.

Garriga, E. y Melé D. (2004). Corporate social responsibility theories: Mapping the territory.Journal of Business Ethics, $53(1-2), 51-71$.

Guédez, V. (2006). Ética y Práctica de la Responsabilidad Social Empresarial: El Aporte de la Empresa al Capital Social. Venezuela: Planeta.

Guerrero, J. C., Cañedo, R., Rubio, R., Samara, M., Cutiño, M. y Fernández, D. J. (2006). Calidad de vida y trabajo. Algunas consideraciones sobre el ambiente laboral de la oficina. ACIMED, 14 (4).

Hellriegel, D., Jackson, S. E. y Slocum Jr., J. W. (2017). Administración: un Enfoque Basado en Competencias. México: Cengage Learning.

Kennedy, P. (1998). A Guide to Econometrics. Fourth Edition. Cambridge MA: MIT press.

Kleinbaum, D. G. y Klein, M. (2010). Logistic Regression. A self\#learning text. Atlanta, GA: Springer

Kotler, P. y Lee N. (2005). Corporate Social Responsibility. Doing the Most Good four Your Copmpany and Your Cause. New Yersey: Wiley

León, F. (2008). La Percepción de la Responsabilidad Social por parte del Consumidor. Visión Gerencial, Universidad de los Andes Venezuela, 1, 83-95 Disponible en: https://www.redalyc.org/pdf/4655/465545878013.pdf Consultado el 5 de diciembre 2019.

López, A. (2013). Hacia la responsabilidad social empresarial de pequeñas empresas: caso México. Revista Internacional Administración \& Finanzas 6(6), 39-54.

Nieto, D. M., Melgar, J. y Coronel, N. A. (2015). Como Medir la Percepción de la Responsabilidad Social Corporativa en la Industria de Dispositivos Médicos. XX Congreso de Internacional de Contaduria, Administración e Informática. UNAM Disponible en http://congreso.investiga.fca.unam.mx/docs/xx/ docs/10.05.pdf Consultado el 5 de diciembre 2019.

Pride, W. M., Hughes, R. J. y Kapoor, J. R. (2015). Introducción a los Negocios México: Cengage Learning.

Randstad. (2015). El ambiente laboral, eso que siempre está y muchas veces no prestamos atención. Randstad. Disponible en: https://www.randstad.com.ar/tendencias-360/archivo/el-ambiente-laboral-eso-que-siempreesta-y-muchas-veces-no-prestamos-atencion_57/ Consultado el 5 de diciembre de 2019.

Raufflet, E., Lozano, J. F., Barrera, D. E. y García, C. (2012). Responsabilidad Social Empresarial. México: Pearson.

Schwartz, P. y Gibb, B. (2000). Cuando las Buenas Compañias se Portan Mal. Barcelona, España: Ediciones Granica

Soto, E. y Cárdenas, J. A. (2007). Ética en las Organizaciones. México: McGraw Hill.

Thompson, A. A., Gamble, J. E. y Peteraf, M. A. (2012). Administración Estratégica: Teoría y Casos. México: McGraw Hill.

Torres, M., Paz, K. y Salazar, F. (2006). Tamaño de una muestra para una Investigación de Mercado. Revista Electrónica Ingeniería Primero. 2, 1-13.

Turker, D. (2009). How corporate social responsibility Influences organizational commitment. Journal of Business Ethics 89(2),189-204.

Copyright 2019 Universidad Autónoma del Estado de México

CC BY-NC-ND 